\title{
Granulomatous interstitial nephritis in a renal allograft
}

\author{
Noriko Nunota $\cdot$ Hirokazu Honda $\cdot$ Takanori Shibata $\cdot$ Osamu Yoshitake \\ Masahiko Murakami · Daisuke Sanada - Akio Yokochi • Noriyuki Kato · \\ Aki Kuroki · Kazuho Honda • Yutaka Yamaguchi · Tetsuzo Sugisaki • \\ Tadao Akizawa
}

Received: 10 September 2011/Accepted: 29 January 2012/Published online: 17 March 2012

(C) Japanese Society of Nephrology 2012

\begin{abstract}
We describe a middle-aged woman in whom granulomatous interstitial nephritis (GIN) developed in a renal allograft. She had undergone bowel resection due to an uncertain diagnosis of active granulomatous bowel disease 30 years earlier. Thereafter, frequent hyperoxaluria as well as calcium oxalate stone and recurrent urinary tract infections had resulted in a progressive deterioration in kidney function over a period of 20 years. She underwent living donor kidney transplantation; however, her kidney function progressively deteriorated, despite transplantation. A biopsy of the renal allograft revealed GIN with granulomatous vasculitis accompanied by calcium oxalate crystals. These as well as the laboratory findings indicated a diagnosis of sarcoidosis. We considered that the aggravated granulomatous inflammation on the allograft was caused by recurrent sarcoidosis accompanied by hyperoxaluria.
\end{abstract}

N. Nunota $\cdot$ H. Honda $(\bowtie) \cdot$ T. Shibata $\cdot$ D. Sanada

A. Yokochi - N. Kato - A. Kuroki - T. Sugisaki - T. Akizawa

Division of Nephrology, Department of Medicine, Showa

University School of Medicine, 1-5-8 Hatanodai, Shinagawa-ku,

Tokyo 142-8666, Japan

e-mail: hondah@med.showa-u.ac.jp

O. Yoshitake · M. Murakami

Division of General and Gastroenterological Surgery,

Department of Surgery, Showa University School of Medicine,

Tokyo, Japan

K. Honda

Department of Pathology, Tokyo Women's Medical University,

Tokyo, Japan

Y. Yamaguchi

Yamaguchi Pathology Laboratory, Chiba, Japan
Keywords Granulomatous interstitial nephritis . Renal allograft $\cdot$ Sarcoidosis $\cdot$ Secondary oxalosis

\section{Introduction}

Granulomatous interstitial nephritis (GIN) is a rare condition that is histologically diagnosed from biopsies of transplanted kidneys [1]. In this setting, drug hypersensitivity and infectious diseases can be the most common etiology of GIN and accurate diagnosis is required, particularly with respect to early post-transplant immunosuppression [2].

Here, we describe GIN in a renal allograft that developed after bowel resection in a patient with active granulomatous bowel disease caused by sarcoidosis. Renal function progressively deteriorated soon after steroid therapy was withdrawn. Aggravation of the granulomatous lesion in the allograft kidney was attributed to sarcoidosis, together with oxalosis and hyperoxaluria after bowel resection.

\section{Case report}

A woman in her early 50s developed end-stage renal disease (ESRD) due to calcium oxalate stone and recurrent urinary tract infections. Approximately $230 \mathrm{~cm}$ of her intestine had been surgically excised 30 years earlier because of active inflammatory bowel disease. The excised intestinal specimen revealed a longitudinal ulcer and straggly granuloma, but did not provide the basis for a definite diagnosis. Hyperoxaluria occurred after bowel resection and calcium oxalate stones often developed. Her kidney function deteriorated over the next 20 years and 

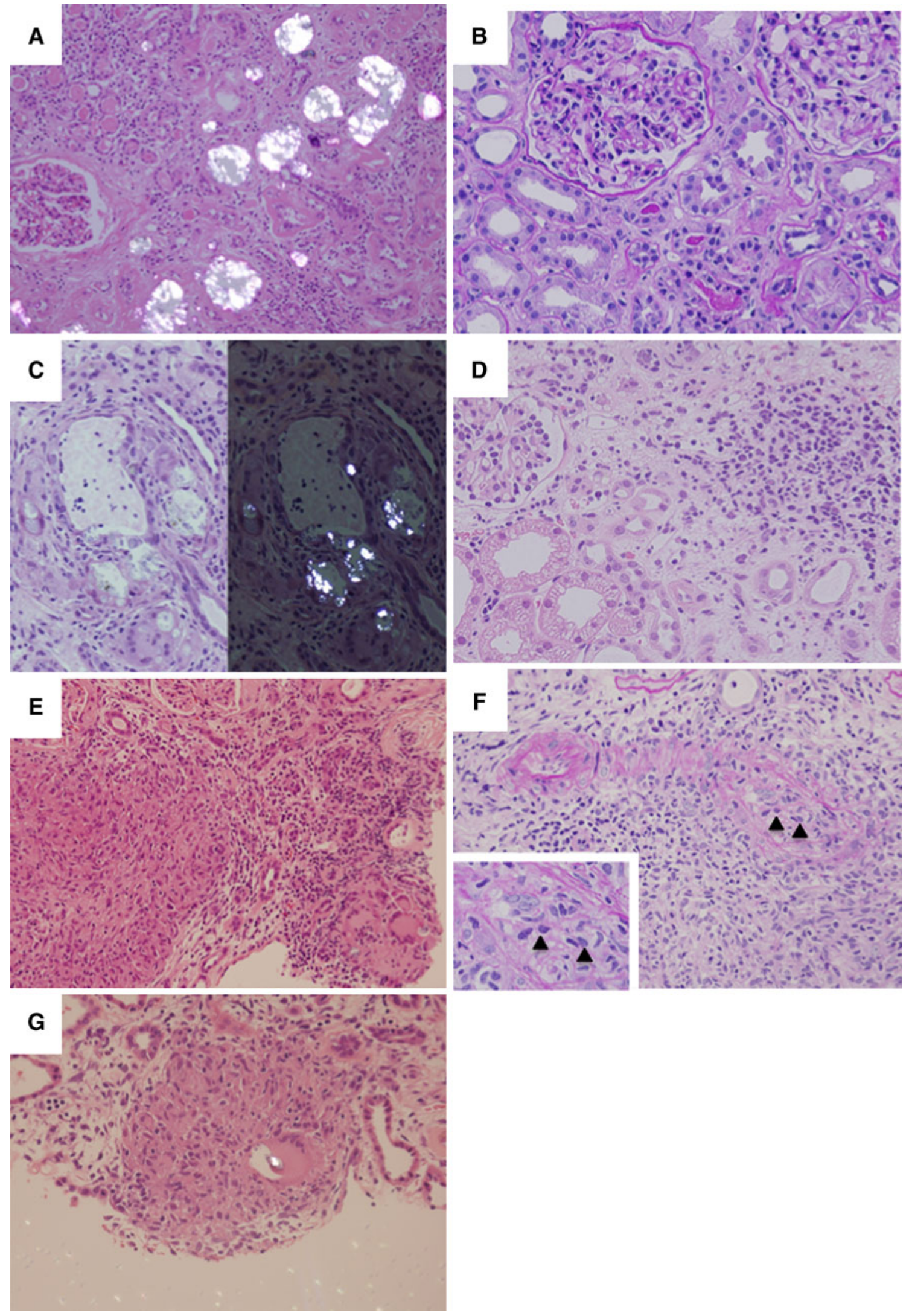
4 Fig. 1 Oxalate crystals in renal tubulointerstitium of the native kidney (polarizing microscopy of H\&E section $\times 20$ ) (a). Mild focal tubulitis at 2 months (PAS stain $\times 40)($ b). Oxalate crystals in renal tubulointerstitium at 6 months (H\&E stain and polarizing microscopy of $\mathrm{H} \& \mathrm{E}$ section $\times 100)(\mathbf{c})$, and focal peritubular capillaritis and lymphocytic aggregation at 11 months $(\mathrm{H} \& \mathrm{E}$ stain $\times 40)($ d). Granulomatous interstitial nephritis with epithelioid cells and giant cells in transplanted kidney at 24 months $(H \& E$ stain $\times 20)($ e). Granulomatous angiitis with epithelioid and mononuclear cells along a small artery that had been invaded by epithelioid cells at 18 months (arrowheads) (PAS stain $\times 40)($ f) and epithelioid granuloma with calcium oxalate crystal at 24 months (polarizing microscopy of $\mathrm{H} \& \mathrm{E}$ section $\times 40)(\mathbf{g})$

hemodialysis was finally started. She underwent living donor kidney transplantation 1 year after starting hemodialysis at our hospital.

Bilateral nephrectomy was performed due to recurrent urinary tract infections before transplantation. Histological analysis (Fig. 1a) of her native kidney revealed multiple birefringent crystalline structures (oxalate crystals) and a few granulomas in the renal interstitium. Computed tomography revealed enlarged mediastinal lymph nodes and lymph nodes around the iliac and inguinal arteries. The intestine was biopsied to assess the activity of inflammatory bowel disease and enlarged inguinal lymph nodes. Non-caseating epithelioid cell granulomas in both specimens suggested systemic granulomatous disease. The laboratory findings before transplantation revealed hemoglobin $8.1 \mathrm{~g} / \mathrm{dL}$, albumin $2.8 \mathrm{~g} / \mathrm{dL}$, creatinine $(\mathrm{Cr}) 10.3 \mathrm{mg} / \mathrm{dL}$, BUN $50.9 \mathrm{mg} / \mathrm{dL}$, calcium adjusted albumin $9.8 \mathrm{mg} / \mathrm{dL}$ (normal range $8.4-10.4 \mathrm{mg} / \mathrm{dl}$ ), phosphate $6.2 \mathrm{mg} / \mathrm{dL}$ (normal range $2.5-4.6 \mathrm{mg} / \mathrm{dl})$, C-reactive protein $<0.2 \mathrm{mg} / \mathrm{dL}$, $25(\mathrm{OH})$ vitamin D $8.0 \mathrm{ng} / \mathrm{dL}$ (normal range $7-41 \mathrm{ng} / \mathrm{mL}$ ), $1,25(\mathrm{OH})_{2}$ vitamin D $12.2 \mathrm{pg} / \mathrm{mL}$ (normal range $20-60 \mathrm{pg} /$
$\mathrm{mL}$ ), angiotensin-converting enzyme 5.7 U/L (normal range 8.3-21.4 U/L), and lysozyme $34.6 \mu \mathrm{g} / \mathrm{mL}$ (normal range $5.0-10.2 \mu \mathrm{g} / \mathrm{mL}$ ). Specific findings of granulomatous diseases including infectious tuberculous or fungal infections, allergic drug reactions, or autoimmune diseases were absent, although systemic inflammatory granulomatous diseases such as sarcoidosis were considered.

The patient underwent renal transplantation followed by standard immunosuppressive therapy comprising methyl prednisolone (mPSL), cyclosporine A (CyA), and mycophenolate mofetil (MMF), and rejection was prevented using CyA and MMF (Fig. 2). The post-transplantation serum $\mathrm{Cr}$ value fell to $1.7 \mathrm{mg} / \mathrm{dL}$ (Fig. 2). However, the serum $\mathrm{Cr}$ increased 2 months later and her kidney function progressively deteriorated (Fig. 2). Renal biopsy findings at 2 months showed mild focal tubulitis, but no evidences of rejection were presented (Fig. 1b). Urinary oxalate levels fluctuated between 15 and $55 \mathrm{mg} /$ day during this period. Renal biopsies repeated at 6 and 11 months revealed mild focal tubulointerstitial nephritis concomitant with oxalate crystals in the tubules (Fig. 1c, d). However, histological assessments of biopsy specimens at 18 and 24 months revealed a granuloma consisting of epithelioid and mononuclear cells around a small artery that had been invaded by epithelioid cells (arrowheads in Fig. 1e) and GIN (Fig. 1f). The granuloma had absorbed a calcium oxalate stone (Fig. 1g). Her levels of serum angiotensinconverting enzyme $(26.7 \mathrm{U} / \mathrm{L})$ and lysozyme $(46.4 \mu \mathrm{g} / \mathrm{mL})$ were elevated and $\mathrm{Ga}^{67}$ uptake by salivary glands was increased at 24 months. We finally diagnosed this systemic granulomatous disease as sarcoidosis with concomitant calcium oxalate crystals.

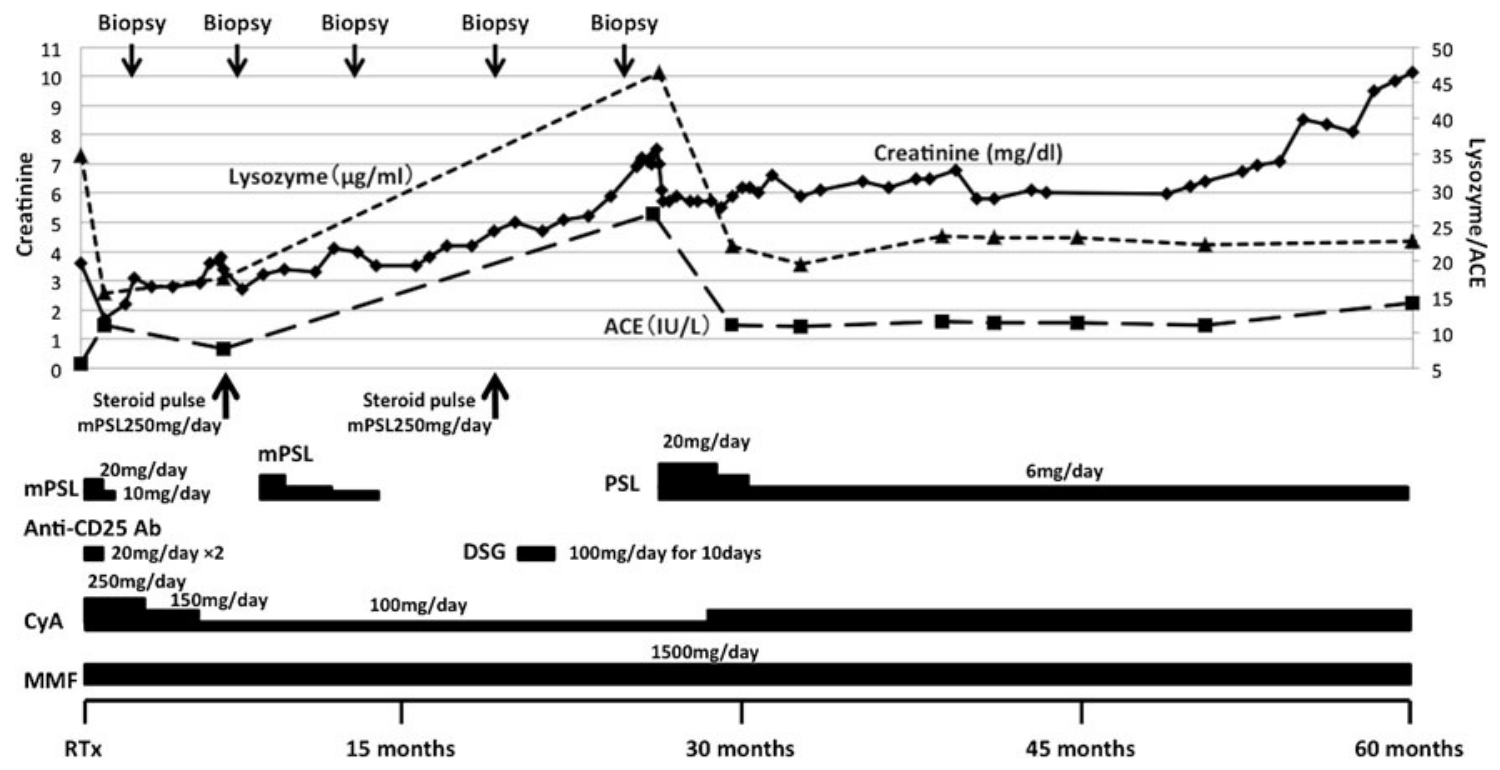

Fig. 2 Clinical course. $D S G$ deoxyspergualin, $C y A$ cyclosporin A, $M M F$ mycophenolate mofetil, $m P S L$ methylprednisolone, $P S L$ prednisolone 
Prednisolone (PSL, $20 \mathrm{mg} /$ day p.o.) and immunosuppressive therapy with $\mathrm{CyA}$ and MMF improved her serum Cr level from 7.5 to $5.7 \mathrm{mg} / \mathrm{dL}$ (Fig. 2). The dosage of PSL was reduced to $6 \mathrm{mg} /$ day over a period of 2 months due to osteonecrosis of the femoral head. The renal graft was lost 5 years after transplantation (Fig. 2).

\section{Discussion}

We described a patient who developed GIN in a renal allograft due to sarcoidosis with concomitant oxalosis. Loss of native kidney function was mainly caused by secondary calcium oxalate stone with a few granulomas. However, massive GIN developed in the allograft kidney and some granulomas absorbed oxalate crystals (Fig. 1g). Vasculitis of the small artery might have been isolated, indicating vascular rejection in the allograft kidney. However, we considered that the vasculitis was associated with sarcoidosis because epithelioid cells had invaded a small artery (Fig. 1e).

Sarcoidosis is a multisystemic disorder of unknown etiology that is characterized by granuloma formation, often in the lung, but it can impact all organs, including the kidney [3]. Autopsy studies indicate that about $20 \%$ of sarcoidosis presents as GIN [4]. Recurrence of sarcoidosis on a transplanted kidney is relatively frequent (27\%) and it often presents with GIN [5]. Hypercalcemia occurs in $10-20 \%$ of patients with sarcoidosis and it is thought to be a consequence of increased intestinal calcium absorption caused by accelerated $1,25(\mathrm{OH})_{2}$ vitamin $\mathrm{D}$ production by activated macrophages [3]. However, our patient did not have hypercalcemia. Although serum $1,25(\mathrm{OH})_{2}$ vitamin D levels were not apparently elevated, the normal serum calcium levels might be explained as restricted calcium absorption by the short length of functional intestine remaining after resection.

We did not initially consider that renal sarcoidosis would present in the allograft kidney, although its function was obviously worsening at 2 months after transplantation. The biopsy specimen of the native kidney did not show typical histological finding(s) of renal sarcoidosis, but the patient might have had sarcoidosis before transplantation, and early post-transplant biopsy findings indicated mild focal tubulointerstitial nephritis. We considered that one of the main reasons for the elevated serum $\mathrm{Cr}$ after transplantation was secondary oxalosis, because oxalate crystals were identified in the allograft kidney at 6 months (Fig. 1c). Thus, immunosuppressive drugs were reduced during the early post-transplant period according to the immunosuppressive regimen (Fig. 2). However, GIN was evident in the allograft at 18 months, when mPSL was withdrawn. This clinical course indicated that sarcoidosis might attack the allograft kidney, even though sarcoidosis did not develop in the native kidney. Thus, immunosuppressive therapy in patients with sarcoidosis who require renal transplantation should be carefully considered.

On the other hand, the reason(s) why granuloma developed in the allograft and appeared to be the cause of its obvious deterioration compared with the native kidney is uncertain. The pathognomonic signs in our patient comprised malabsorption-related oxalosis and hyperoxaluria. Since plasma oxalate levels are significantly increased in patients with ESRD, renal oxalate excretion is excessive in patients after kidney transplantation [6]. Hyperoxaluria might lead to oxalate deposits in allograft tissue and finally lead to renal insufficiency [6, 7]. Moreover, oxalate might be associated with granulomatous lesions in the kidney and be attributable to systemic granulomatous disease, as oxalate deposition appears to be endogenously derived [8]. Oxalates are notably found in granulomas in various inflammatory diseases, including sarcoidosis [9, 10]. Therefore, excessive allograft oxalate excretion could not only cause granuloma, but might also be associated with the formation of granulomas in sarcoidosis.

\section{References}

1. Meehan SM, Josephson MA, Haas M. Granulomatous tubulointerstitial nephritis in the renal allograft. Am J Kidney Dis. 2000; 36:E27.

2. Lapasia JB, Kambham N, Busque S, Tan JC. Renal allograft granulomas in the early post-transplant period. NDT Plus. 2010;3:397-401.

3. Dahl K, Canetta PA, D’Agati VD, Radhakrishnan J. A 56-yearold woman with sarcoidosis and acute renal failure. Kidney Int. 2008;74:817-21.

4. Sheffield EA. Pathology of sarcoidosis. Clin Chest Med. 1997; 18:741-54.

5. Aouizerate J, Matignon M, Kamar N, Thervet E, Randoux C, Moulin B, et al. Renal transplantation in patients with sarcoidosis: a French multicenter study. Clin J Am Soc Nephrol. 2010; 5:2101-8.

6. Bagnasco SM, Mohammed BS, Mani H, Gandolfo MT, Haas M, Racusen LC, et al. Oxalate deposits in biopsies from native and transplanted kidneys, and impact on graft function. Nephrol Dial Transplant. 2009;24:1319-25.

7. Elgstoen KB, Johnsen LF, Woldseth B, Morkrid L, Hartmann A. Plasma oxalate following kidney transplantation in patients without primary hyperoxaluria. Nephrol Dial Transplant. 2010;25:2341-5.

8. Hoppe B, Beck BB, Milliner DS. The primary hyperoxalurias. Kidney Int. 2009;75:1264-71.

9. Symmans PJ, Brady K, Keen CE. Calcium oxalate crystal deposition in epithelioid histiocytes of granulomatous lymphadenitis: analysis by light and electronmicroscopy. Histopathology. 1995;27:423-9.

10. Ghio AJ, Roggli VL, Kennedy TP, Piantadosi CA. Calcium oxalate and iron accumulation in sarcoidosis. Sarcoidosis Vasc Diffuse Lung Dis. 2000;17:140-50. 